\title{
A study on Ovine pneumonic pasteurellosis: Isolation and Identification of Pasteurellae and their antibiogram susceptibility pattern in Haramaya District, Eastern Hararghe, Ethiopia
}

\author{
Haimanot D Marru ${ }^{1 *}$, Takele T Anijajo² and Adem A Hassen ${ }^{3}$
}

\begin{abstract}
Background: Sheep constitute the second major component of livestock in Ethiopia. However, efficient utilization of this potential resource is hampered by combination of health problems, poor management and feed shortage. Haramaya district is one of the remote settings in Ethiopia where information about the livestock disease is not well documented. Hence this study was conducted to determine the causative agents and their antimicrobial susceptibility pattern of bacterial Pasteurella isolates among pneumonic ovine in Haramaya district, Eastern Hararghe, Ethiopia.

Results: Out of 256 samples examined, Pasterurella was isolated in 64 (25\%), of which 38 (59.4\%) were from lungs and $26(40.6 \%)$ were from nasal cavities. $87.5 \%$ of the isolates were Mannheimia haemolytica and $12.5 \%$ were Pasteurella multocida. All of the isolates from the lungs were Mannheimia haemolytica whereas $69 \%$ of the isolates from nasals cavities were Mannheimia haemolytica. Age and body temperature were significantly associated with Pasteurella isolates from clinic $(P<0.05)$. Despite diverse in the site of origins, the isolates exhibited uniformity in sensitivity to a majority of the antibacterial agents. The most effective drug was Cholramphenicol (100\%) followed by Sulfamethoxazole (89.1\%) and Tetracycline (84.4\%). Both species were completely resistant to Gentamycin and Vancomycin.
\end{abstract}

Conclusion: Mannheimia haemolytica is the most common cause of ovine pneumonic pasteurellosis in the study area. The isolates were susceptible to limited antimicrobial agents. Therefore, the antimicrobial susceptibility test should be conducted before treatment, except for critical cases.

Keywords: Antimicrobial, Haramaya district, Hararghe, Bacterial isolation and identification, Pneumonic pasteurellossis

\section{Background}

Pneumonia is extremely common in sheep and can be responsible for enormous financial losses worldwide. The condition usually appears when sheep are exposed to combinations of predisposing factors such as adverse physical condition, physiological stress, bacterial and viral infections. As the exact nature of these combinations is unknown, much remain to be understood about

\footnotetext{
* Correspondence: haimadis2012@gmail.com

${ }^{1}$ College of Health Sciences, School of Veterinary Medicine, Wollega University, P. O. Box 395, Nekemte, Ethiopia

Full list of author information is available at the end of the article
}

why the disease occurs in the way it does [1]. SubSaharan Africa is endowed with a large population of sheep. The population of sheep is estimated at 132.5 million head [2]. However, animal productivity per head is low.

Ethiopia lies within the tropical latitude of Africa and has an extremely diverse topography, a wide range of climatic features and a multitude of agro-ecological zone which makes the country suitable for different agricultural production system. This in turn has contributed to the existence of a large diversity of farm animal genetic resource in the country [3]. Sheep constitute the

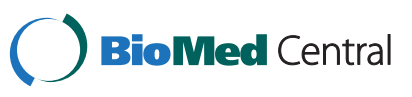


second major component of livestock in Ethiopia. Despite the large livestock population of Ethiopia the economic benefits remain marginal due to prevailing diseases, poor nutrition, poor animal production systems, reproductive inefficiency management constraint and general lack of Veterinary core [4].

Pneumonic pasteurellosis is one of the priority diseases that deserve control. However, control of pneumonic pasteurellosis is difficult task that requires integration of various techniques. Developed and developing countries practice various control mechanisms for primary diseases. While developing countries including Ethiopia could not apply the strategies used by developed countries because of economic reason. The most economic and feasible control method for developing nations is the use of vaccine (Fekadu and Girum: Study of the prevalent serotype of Ovine pasteurellosis in the high land of Eastern Amhara sub-region. Kombolcha Veterinary laboratory, unpublished: 2001).

In Ethiopia there are some works conducted on ovine pneumonic pasteurellosis: seroprevalence, bacteriological prevalence in pneumonic lungs and detection of most prevalent serotyping throughout the selected sites of Ethiopia. But, no work was done in Eastern Hararghe at Haramaya district on the Pasteurella isolation as well as the drugs or antimicrobial to which these isolates are susceptible for. Therefore, this study was focused on the isolation and identification of Pastuerella species that involved in ovine pneumonic pasteurellosis among apparently healthy sheep with pneumonic lungs slaughtered at Haramaya municipal abattoir and sheep with clinical manifestation presented to the Haramaya veterinary clinic during the study period. In addition, this study attempted to determine drug susceptibility pattern of these isolates.

\section{Methods \\ Study area}

The study was conducted at Haramaya district which is located in Eastern Hararghe Zone of Ethiopia, approximately $500 \mathrm{kms}$ East of Addis Ababa. Its elevation is approximately $2000 \mathrm{~m}$ a.s.l. and the mean annual temperature and relative humidity are $18^{\circ} \mathrm{C}$ and $65 \%$, respectively. An annual rainfall is approximately $900 \mathrm{~mm}$, with a bimodal distribution pattern, peaking in mid April and mid August. There are four seasons, such as a short rainy season (from mid March to mid May), a short dry season (from end May to June), a long wet season (July to mid October) and a long dry season (end of October to February). Main pasture production is expected after the short rainy season, continuing until the end of the long wet season. Mixed type agriculture is the main occupation of the population of the area. According to the veterinary sector of Haramaya district agricultural office 2000 report, a total livestock population of this district was 193,334; of which 64,510 Cattle, 28,359 goats, 18,930 sheep, 15,277 donkeys, 5 mules, 530 camels and 65,723 poultry [5].

\section{Study design}

A cross-sectional study was undertaken from November 2007-April 2008 on ovine pneumonic pasteurellosis to isolate and identify the Pasteurella species from specimens of nasal swab of clinically suspected pneumonic sheep from Haramaya veterinary clinic and lung swab of pneumonic lungs of apparently healthy sheep slaughtered at Haramaya municipal abattoir.

\section{Study animal}

The animals used in this study were apparently healthy sheep slaughtered at Haramaya municipal abattoir and sheep with clinical manifestations presented to the Haramaya veterinary clinic during the study period. At the abattoir, the lungs of the slaughtered animals were visualized, palpated and inspected thoroughly and finally the pneumonic lungs were considered. According to the postmortem meat inspection principle the lungs of the slaughtered animal were visualized and palpated for haemorrhage, edema and pneumonia. Up on visualization, palpation and inspection the lungs with consolidated inflamed area, deep red and sharply demarcated lesion were considered as pneumonic lungs [6]. All sheep irrespective of age, sex, and color were examined at clinic for the evidence of pneumonic pasteurellosis. Up on clinical examination, all sheep manifesting; anorexia, coughing, dyspnea, lethargy, serous to muco-purulent ocular and nasal discharge, and fever were considered. In both cases the animals with pneumonic lung and the mentioned clinical features were considered to be study population. The sample size for each varied according to the availability of suspected cases of sheep with pneumonia in respective to study site. A total of 256 (83 from clinic and 173 from abattoir) suspected cases were collected.

\section{Ethical consideration}

Investigators treated animals with kindness and took proper care by minimizing discomfort, distress or pain. They assumed that all procedures which would cause pain in human beings may cause pain in study animals. The required procedures were conducted by qualified and experienced persons [7]. The ethical clearance was obtained from Haramaya University ethical review board.

\section{Data collection \\ Nasal swab}

Each animal was individually identified and restrained by an assistant and kept fixed. After disinfection of external part of the nose with $70 \%$ alcohol, a sterile cotton-tipped swab was inserted in to the nostril and rotated against 
the wall of the nasal cavity [8]. The swab was placed in labeled sterile test tube that contains $3 \mathrm{ml}$ of tryptose Soya broth, and then kept in an ice box for transport to Haramaya University, FVM. Laboratory [8].

\section{Lung swab}

Following the slaughter of the apparently healthy animal, all the lungs were inspected according to the standard postmortem meat inspection procedure. Up on inspection the surface of each suspected lung was incised using sterile scalpel blade and the inner surface of the incision was sampled with sterile swab. The swab was transported to Veterinary Microbiology Laboratory of Haramaya University in the same procedure with nasal swab [9].

\section{Isolation and Identification of Pasteurella}

The isolation and identification of Pasteurella were performed at the Veterinary Microbiology Laboratory of Haramaya University using techniques recommended by Hardy Diagnostics, Santa Maria, CA, USA. The isolation and identification involves the following steps: first, the pre-enriched in tryptose Soya broth specimen was incubated for $24 \mathrm{hrs}$ at $37^{\circ} \mathrm{C}$. After 24 hrs incubation, a loop full of the broth cultures were taken and streaked over an identified Petri-plate containing blood agar base supplemented with $7 \%$ sheep blood and immediately incubated aerobically at $37^{\circ} \mathrm{C}$ for 24 hours [9]. Secondly, from culture positive plates, typical colonies were subjected to gram's staining to study staining reactions and cellular morphology under light microscope, at 100x magnification. Mixed and gram-negative bacteria were further sub cultured with due care, on both blood and MacConkey agar plates [9] for further analysis. The growth of typical colonies on both blood and MacConkey agar was characterized using blood agar for the presence of haemolysis, the type of haemolysis, the general appearance of colonies (morphology, color, shape size and consistency) and the ability to ferment lactose [10]. Thirdly, pure cultures of single colony type from both Blood and MacConkey agars were transferred onto nutrient agar-slants for a series of primary biochemical tests: catalase (Hydrogen peroxide, Fisher Chemical, UK), oxidase (TM-pphenylenediamine dihydrocholoide, Merck Co., Germany) and fermentative/ oxidative (OF Basal Medium, Titan Biotech Ltd, India). Final identification of the bacteria to the species level was aided by using the secondary tests which include: metabolism of sugars such as glucose and L-arabinose; and alcohols and tests for metabolic end products such as Indole (Peptone water, Merck Co., Germany) following standard procedures $[11,12]$. The assay for biochemical properties of the bacterial isolates were conducted according to MacFaddin's method [13]. For reliable identification and comparison of results, the AIPE 20 system
(Biomariux France) was used. If the organism is able to produce a narrow zone of haemolysis on Blood agar and grow on McConkey agar, but unable produce indole, interpreted as P.haemolytica. If the organism is unable to produce haemolysis on Blood agar and cannot grow on MacConkey, but able to produce indole, interpreted as P. multocida.

\section{Antimicrobial susceptibility test}

The antimicrobial susceptibility test on the isolates was performed according to the National Committee for Clinical Laboratory Standards (NCCLS, 1990) [14] method using Kibry-Bauer disk diffusion test on Muller-Hinton agar (Oxoid CM0337 Basingstoke, England). Escherichia coli ATCC 25922 was used as a quality control organism for the antimicrobial susceptibility test [15]. The isolates were tested for the following antibiotics; Chloramphenicol (CAF) 30Ng, Tetracycline (TTC) 30Ng, Penicillin-G (P) 10 unit, Ampicilin (Am) 10Ng, Sulfamethoxazole(SxT) $5 \mathrm{Ng}$, Streptomycin(S) $10 \mathrm{Ng}$, Vancomycin (VA) $30 \mathrm{Ng}$ and Gentamicin $(\mathrm{CN}) 30 \mathrm{Ng}$ based on the procedure recommended by Carter [16] and Quinn et al. [17]. The zone of inhibition was interpreted based on the Performance Standards for Antimicrobial Susceptibility Testing; Sixteenth Informational Supplement [18] as detailed in Table 1.

\section{Data entry and analysis}

Data collected from both the clinic and abattoir were recorded in the format developed for this purpose and later on entered into the Microsoft excel 2000 program and analyzed using STATA 7.0 software. Association of host risk factors with swab culture positives was calculated. A statistically significant association between variables was considered to exist if the computed $\mathrm{p}$-valve was less than 0.05 .

Table 1 Zone interpretive chart for antimicrobials (inhibition zone diameter in $\mathrm{mm}$ )

\begin{tabular}{lcccc}
\hline $\begin{array}{c}\text { Antimicrobial } \\
\text { agent }\end{array}$ & $\begin{array}{c}\text { Disc } \\
\text { potency } \\
(\boldsymbol{\mu g})\end{array}$ & $\begin{array}{c}\text { Resistant } \\
(\mathbf{s})\end{array}$ & Intermediate & Susceptible \\
\hline Tetracycline & 30 & 14 & $15-18$ & $\mathbf{( \geq )}$ \\
Streptomycin & 10 & 11 & $12-14$ & 15 \\
Chloramphenicol & 30 & 12 & $13-17$ & 18 \\
Vancomycin & 30 & 14 & $15-16$ & 17 \\
Penicillin-G & $10 \mathrm{u}$ & 28 & - & 29 \\
Ampicillin & 10 & 13 & $14-16$ & 17 \\
Sulphamethoxozole & 25 & 10 & $11-15$ & 16 \\
Gentamicine & 10 & 12 & $13-14$ & 15 \\
\hline
\end{tabular}

Source: Clinical and Laboratory Standards Institute (CLSI) [18]. 


\section{Results}

\section{Bacterial isolation}

From 256 samples (83 nasal swabs and 173 lung swab) collected and cultured, Pasteurella was isolated successfully in 64 (25\%) sheep. Out of 64, 26 (40.6\%) were from nasal cavities and 38 (59.4\%) from lungs. Eighty seven point five percent of the isolate was $M$. haemolytica and 12.5\% was P.multocida and of the lung samples which were culture positive, $100 \%$ was $M$. haemolytica. In nasal cavities, of the samples which were culture positive, $69 \%$ of the isolate was M. haemolytica and $31 \%$ of the isolate was P.multocida (Table 2). On the basis of these results $M$. haemolytica was the most common cause of pneumonic pasteurellosis in sheep at Haramaya district.

$M$. haemolytica was isolated in both clinical and abattoir, but P.multocida was exclusive to clinical cases. The magnitude of $M$. haemolytica was almost equal in both clinical (21.69\%) and abattoir (21.96\%) cases; however, the overall magnitude of the bacteriological confirmed cases is higher in clinical cases (31.3\%) than abattoir cases $(21.96 \%)$. The result indicated that, there is positive association between body temperatures of the animals at clinic and bacteriological confirmed cases of pneumonic pasteurellosis $(\mathrm{P}=0.007)$, but age has negatively associated $(\mathrm{P}=0.028)$ particularly with $P$.multocida $(\mathrm{P}=0.004)$ infection and sex has no any association with both species $(\mathrm{P}>0.05)$ (Table 3$)$. No associations were observed between the risk factors and pneumonic pasteurellosis at abattoir $(\mathrm{P}>0.05)$ (Table 4$)$.

\section{Antimicrobial susceptibility pattern}

Sixty four isolates of Pasturella isolates from clinic and abattoir were subjected to a panel of eight antimicrobials. The antimicrobial susceptibility pattern of the isolates indicated that all isolates were 100\%, 100\%, 90.6\%, and $87.5 \%$ resistant to gentamycin, vancomycin, streptomycin and penicillin-G respectively. On the other hand, the isolates were $100 \%, 89.1 \%, 84.4 \%$ and $53.1 \%$ sensitive to chloramphenicol, sulfamethoxazole, tetracycline and ampicilin respectively (Table 5). Despite diverse in the site of origins, the isolates exhibited uniformity in sensitivity to a majority of the antibacterial agents. M. haemolytica showed $100 \%$ resistance to gentamycin and vancomycin while they were $100 \%$ sensitive to chloramphenicol followed by $89.3 \%$ and $83.9 \%$ to sulfamethoxazole and tetracycline consequently. Similarly P.multocida showed $100 \%$ sensitivity to chloramphenicol followed by sulfamethoxazole and tetracycline. However, P. multocida is more susceptible to sulfametrioxazole and tetracycline, but not statically significant $(\mathrm{P}>0.05)$.

\section{Discussion}

In the present study, Pasteurella species were isolated in $25 \%$ of the sheep, of which $59.4 \%$ and $40.6 \%$ were contributed from abattoir and clinic respectively. M. haemolytica was accounted for $87.5 \%$. The prevalence of $M$. haemolytica was almost similar both at abattoir (21.96\%) and clinic (21.69\%). P. multiocida was discovered only from nasal swabs. Pneumonic pasteurellesis was positively associated with body temperature and negatively associated with the age of suspected ovine particularly P. multiocida. Sex has no any association.

In the present study, M. haemolytica was isolated from $21.96 \%$ of lung samples examined. This result is greater than the findings of Eshetu [19] and Nurhusien [20], which were reported as $13 \%$ and $8.7 \%$ respectively, but lower, than the findings of Mohammed [21] and Aschalew [22] that were reported as $40.8 \%$ and $56 \%$ respectively in pneumonic lungs. In nasal swab $M$. haemolytica was discovered with the rate of $21.7 \%$, which is similar with that of the lung swab. Nonetheless, the present result indicates high prevalence of the isolate than the work done by Eshetu [19] who reported 13\% in the same area before sixteen years ago. This difference might be due to the type

Table 2 Pasturella Isolates from nasal cavity and lung samples of Ovine at clinic and abattoir at Haramaya district in Eastern Hararghe, Ethiopia

\begin{tabular}{|c|c|c|c|c|c|}
\hline \multirow[t]{2}{*}{ Site } & \multicolumn{2}{|c|}{ Species } & \multirow[t]{2}{*}{ Total } & \multirow{2}{*}{$\begin{array}{l}\text { Percentage } \\
\text { of total } \\
\text { isolates }\end{array}$} & \multirow[t]{2}{*}{ X2 (P-value) } \\
\hline & $\begin{array}{c}\text { M.haemolytica } \\
\text { Positive }\end{array}$ & $\begin{array}{c}\text { P.multocida } \\
\text { Positive }\end{array}$ & & & \\
\hline \multicolumn{6}{|l|}{ Nasal cavity } \\
\hline Positive & $18(69 \%)$ & $8(31 \%)$ & $26(31.3 \%)$ & $26(40.6 \%)$ & \\
\hline Negative & & & $57(68.7 \%)$ & & \\
\hline Total & & & $83(100 \%)$ & & $2.6208(0.105)$ \\
\hline \multicolumn{6}{|l|}{ Lung sample } \\
\hline Positive & $38(100 \%)$ & - & $38(21.96)$ & $38(59.4 \%)$ & \\
\hline Negative & & & 135(78.04\%) & & \\
\hline Total & & & $173(100 \%)$ & & \\
\hline Total & $56(87.5 \%)$ & $8(12.5 \%)$ & $256(100 \%)$ & $64(25 \%)$ & \\
\hline
\end{tabular}


Table 3 Association of culture positive results with the risk factors (age, sex and body temperature) from clinic cases at Haramaya district in Eastern Hararghe, Ethiopia

\begin{tabular}{|c|c|c|c|c|c|c|c|c|}
\hline Age & Young & Adult & & Total & & $C h i^{2}$ & P-value & Overall \\
\hline \multicolumn{9}{|c|}{ M. haemolytica } \\
\hline Negative & 17 & 48 & & 65 & & 2.2405 & 0.134 & \\
\hline Positive & 8 & 10 & & 18 & & & & \\
\hline Total & 25 & 58 & & 83 & & & & $C h i^{2}=4.8501 P=0.028$ \\
\hline \multicolumn{9}{|l|}{ P.multocida } \\
\hline Negative & 19 & 56 & & 75 & & 8.472 & 0.004 & \\
\hline Positive & 6 & 2 & & 8 & & & & \\
\hline Total & 25 & 58 & & 83 & & & & \\
\hline Sex & Male & Female & & Total & & $\mathrm{Chi}^{2}$ & P-value & \\
\hline \multicolumn{9}{|c|}{ M.haemolytica } \\
\hline Negative & 40 & 25 & & 65 & & 2.9443 & 0.086 & \\
\hline Positive & 7 & 11 & & 18 & & & & \\
\hline Total & 47 & 36 & & 83 & & & & Chi2 $=2.6186 \operatorname{Pr}=0.106$ \\
\hline \multicolumn{9}{|l|}{ P.multocida } \\
\hline Negative & 44 & 31 & & 75 & & 1.3187 & 0.251 & \\
\hline Positive & 3 & 5 & & 8 & & & & \\
\hline Total & 47 & 36 & & 83 & & & & \\
\hline Temprature & $38.5-39.50^{c}$ & $39.6-400^{c}$ & $>400^{c}$ & & Total & & & \multirow{4}{*}{$C h i^{2}=10.0614 p=0.007$} \\
\hline Negative & 21 & 23 & 13 & & 57 & & & \\
\hline Positive & 4 & 7 & 15 & & 26 & & & \\
\hline Total & 25 & 30 & 28 & & 83 & & & \\
\hline
\end{tabular}

Table 4 Association of culture positive results from abattoir with risk factors (age and sex) in Abattoir cases at Haramaya District in Eastern Hararghe, Ethiopia

\begin{tabular}{|c|c|c|c|c|c|}
\hline Age & Young & Adult & Total & $C h i^{2}$ & P-value \\
\hline \multicolumn{6}{|l|}{ M.haemolytica } \\
\hline Negative & 12 & 123 & 135 & 2.7560 & 0.097 \\
\hline Positive & 7 & 31 & 38 & & \\
\hline Total & 19 & 154 & 173 & & \\
\hline \multicolumn{6}{|l|}{ P.multocida } \\
\hline Negative & 19 & 154 & 173 & - & - \\
\hline Positive & - & - & - & & \\
\hline Total & 19 & 154 & 173 & & \\
\hline Sex & Male & Female & Total & $\mathrm{Chi}^{2}$ & P-value \\
\hline \multicolumn{6}{|l|}{ M.haemolytica } \\
\hline Negative & 118 & 17 & 135 & 1.1348 & 0.567 \\
\hline Positive & 31 & 7 & 38 & & \\
\hline Total & 149 & 24 & 173 & & \\
\hline \multicolumn{6}{|l|}{ P.multocida } \\
\hline Negative & 149 & 24 & 173 & - & - \\
\hline Positive & - & - & - & & \\
\hline Total & 149 & 24 & 173 & & \\
\hline
\end{tabular}

of sample taken from purely pneumonic lung in the present study. The other possible explanation may be improvement of the health facilities within the last $\sim$ two decades.

Comparing the two Pasteurella spp, M. haemolytica constituted $87.5 \%$ of the total indicated that, M. haemolytica was the major causative agent involved in ovine pneumonic pasteurellosis. This is consistent with previous reports of Aschalew [22], Eshetu [19], Mohammed [21], and Tesfaye [23]. M.haemolytica, which is a normal flora of the upper respiratory tract, may play a secondary role after the primary initiating agent suppressed the host defense mechanism, and favors the multiplication of Pasteurella species leading to bronchopneumonia in purely pneumonic animal [24]. Although the percentage isolation was relatively low (12.5\%), the possible role of P. multocida in the a etiology and pathogenesis of ovine pneumonia should not be under estimated.

In our study Paseurella species were isolated in 31.3\% of nasal swabs. Although they may be found occasionally as a normal inhabitant of the respiratory system, experimental evidence has shown that under certain conditions associated with debilitation, nutrition and climatic factors, these organisms may singly or in concert with other organisms flare up to cause severe infections with 
Table 5 Antimicrobial susceptibility pattern of Pasturella Isolates from nasal cavity and lung samples of Ovine at clinic and abattoir at Haramaya district in Eastern Hararghe, Ethiopia

\begin{tabular}{|c|c|c|c|}
\hline \multirow{2}{*}{$\begin{array}{c}\text { Antimicrobials } \\
\text { tested }\end{array}$} & \multicolumn{2}{|c|}{ Species of bacteria } & \multirow[t]{2}{*}{ Total } \\
\hline & M.haemolytica & P.multocida & \\
\hline \multicolumn{4}{|l|}{ Ampicllin } \\
\hline Sensitive & $30(53.6 \%)$ & $4(50 \%)$ & $34(53.1 \%)$ \\
\hline Resistance & $26(46.4 \%)$ & $4(50 \%)$ & $30(46.8 \%)$ \\
\hline \multicolumn{4}{|l|}{ Chloramphenicol } \\
\hline Sensitive & $56(100 \%)$ & $8(100 \%)$ & $64(100 \%)$ \\
\hline Resistance & - & - & - \\
\hline \multicolumn{4}{|l|}{ Gentamycin } \\
\hline Sensitive & $0(0 \%)$ & - & - \\
\hline Resistance & $56(100)$ & $8(100 \%)$ & $64(100 \%)$ \\
\hline \multicolumn{4}{|l|}{ Tetracycline } \\
\hline Sensitive & 47(83.9\%) & $7(87.5 \%)$ & $54(84.4 \%)$ \\
\hline Resistance & $9(16.1 \%)$ & $1(12.5 \%)$ & $10(15.6 \%)$ \\
\hline \multicolumn{4}{|l|}{ Penicillin-G } \\
\hline Sensitive & $6(10.7 \%)$ & $2(25 \%)$ & $8(12.5 \%)$ \\
\hline Resistance & $50(89.3 \%)$ & $6(75 \%)$ & $56(87.5 \%)$ \\
\hline \multicolumn{4}{|l|}{ Streptomycin } \\
\hline Sensitive & $5(8.9 \%)$ & $1(12.5 \%)$ & $6(9.4 \%)$ \\
\hline Resistance & $51(91.1 \%)$ & $7(87.5 \%)$ & $58(90.6 \%)$ \\
\hline \multicolumn{4}{|l|}{ Sulfamethoxazole } \\
\hline Sensitive & $50(89.3 \%)$ & $7(87.5 \%)$ & 57(89.1\%) \\
\hline Resistance & $6(10.7 \%)$ & $1(12.5 \%)$ & $7(10.9 \%)$ \\
\hline \multicolumn{4}{|l|}{ Vancomycin } \\
\hline Sensitive & - & - & - \\
\hline Resistance & $56(100 \%)$ & $8(100)$ & 64(100\%) \\
\hline
\end{tabular}

high morbidity and mortality. In this particular study the recruitment of animals was based on their definitive clinical sign and symptom to pneumonia and the identification of Pateurella species among these animals makes more soundable and interesting. The magnitude of $M$. haemolytica was almost equal in nasal swabs (21.69\%) and lung swabs (21.96\%). P. multiocida was not isolated in the lung samples in the present study, but it was discovered in nasal swab at the rate of $9.6 \%$. This result, however, supports the idea of previous studies $[21,25]$ that reported $P$. multocida from respiratory tract of sheep and goats with very lower isolation rates from lung.

In the current study a significant association between pneumonic pasteurellesis and body temperature of suspected ovine irrespective of Pasteurella species was observed. This result coincides with the findings of (Fekadu and Girum: Study of the prevalent serotype of Ovine pasteurellosis in the high land of Eastern Amhara subregion. Kombolcha Veterinary laboratory, unpublished:
2001) that explained, pneumonic pasteurellosis is characterized by fever, driving or pushing of the body as result of forced respiration, dry coughing and muco-purulent nasal discharges. Similarly, the disease is significantly associated with the age of sheep. This result is also in agreement with findings of Gilmour and Gilmour [26], that elucidates pneumonic pasteurellosis occur in all ages of sheep and goats, with the most susceptible in lambs and kids during first life, and dams at lambing.

It is important to monitor the antimicrobial susceptibility of Pasteurella species to determine resistance development. Increase in resistance against antibiotics in both $P$. multocida and $M$. haemolytica isolates have been reported $[27,28]$. In our study, according to the antimicrobial susceptibility test results, chloramphenicol (100\%), sulfamethoxazole (89.1\%) and tetracycline (84.4\%) were the most effective drugs, whereas ampicilin (53.1\%) was the only intermediate drug. Penicillin-G (12.5\%) and streptomycin (9.4\%) were inefficient drugs; gentamycin and vancomycin were totally inactive against both isolates. This was in line with the literature which stated as chloramphenicol is highly effective and well-tolerated broad spectrum antibiotic to many genera of gram-positive and gram-negative bacteria [24]. Susceptibility to sulfamethoxazole over $80 \%$ was very close to the rate reported previously [28]. However, this result contradicts the findings of Aschalew [22] who reported that tetracycline as ineffective drug of choice. This difference strengthens the recommendation of Kaan et al. [29] which stated that "Antibiotic susceptibility profiles of $P$. multocida and $M$. haemolytica help veterinarians to choose appropriate antibiotic against bovine respiratory disease; however, antibiotic susceptibility studies should be renewed periodically". In addition, pre-existing resistances in which the cellular mechanisms required for antimicrobial susceptibility are absent from the bacterial cell or acquired genetically because of chromosomal mutation and accusation of transferable genetic material. Treatment with a specific antimicrobial agent selects those micro-organisms that have pre-existing or acquired resistance [9].

One of the interesting findings of our study was the demonstration of the highest resistance of Pasteurella isolates against gentamycin (100\%) and vancomycin (100\%). In contrast, Esra et al. [30], reported that gentamycin (95.0\%) as the most effective antibiotic against M. haemolytica isolates. Similarly Post et al. [31], reported $90.0 \%$ of M. haemolytica isolates revealed from cattle with bovine respiratory disease complex were markedly susceptible to gentamycin. This might be due to difference in the strain of the isolate that may cause pasteurellosis in different species of animals or due to the existence of host factors that may affect the action of drug in sheep. However this study strengthens the statement vancomycin is 
active against most gram-positive bacteria, but is not effective against gram-negative cells because of their large size and poor penetrability [28]. In this study P. multocida showed resistance to penicillin-G, this result is in contrary to literature [32] which indicates most strains of P. multocida are susceptible to penicillin-G.

Limitation of the study: this study was conducted in short period of time; hence we did not saw the seasonal variation of the isolates. In addition, even though identification of serotypes is so important, in our study it was not conducted due to lack of facilities in our laboratory. A part from these limitations our study has the following strengths: the sample was collected from both abattoir and clinic which shows the reliability of the data, the isolates were obtained from pneumonic ovine that made us compare the clinical case with ethological agents, all laboratory works were followed standard procedures and quality control was exhibited in each step of the work.

\section{Conclusions}

In conclusion, Pneumonic Pasteurellosis was the major disease of sheep in the area and $M$. haemolytica is the most common cause. Being young animal was a risk factor for the disease. Both species were susceptible to limited antimicrobial agents. Chloramphenicol, sulfamethoxazole and tetracycline were effective drugs whereas gentamycin and vancomycin were totally inactive against the isolates. Measures such as, improving management practices by providing optimal sanitation and air quality in housing, minimizing transportation stress, providing good quality hay and water, and supplement as appropriate should be taken into account to reduce the prevalence. In this line bacterial isolation and antibiotic susceptibility test should be conducted before treating with antibiotics except for critical ones. Moreover, further serotypoing and molecular techniques are needed to identify the isolate to the strain level.

\section{Competing interests}

All authors have declared that no competing interests exist.

\begin{abstract}
Authors' contributions
HD: Conception of the research idea, designing and data collection, data analysis and interpretation, and manuscript reviewing. TT: Data collection, interpretation of the results, and drafting the manuscript. (AA): Data collection, interpretation of the results and drafting the manuscript with $\Pi$. All authors read and approved the final manuscript.
\end{abstract}

\section{Acknowledgements}

We are very much grateful to the inhabitants of all staff members of Haramaya University, Facility of Veterinary Medicine and Haramaya district veterinary clinic and abattoir workers, for their co-operation in different aspects of the study without them the study wouldn't be possible. We thank Haramaya University Facility of Veterinary Medicine, for partial funding of the project.

\section{Author details}

${ }^{1}$ College of Health Sciences, School of Veterinary Medicine, Wollega University, P. O. Box 395, Nekemte, Ethiopia. ${ }^{2}$ School of Biomedical and
Laboratory Sciences, College of Medicine and Health Sciences, University of Gondar, P.O. Box 196, Gondar, Ethiopia. ${ }^{3}$ Faculity of Veterinary Medicine, Haramaya University, P. O. Box 138, Dire Dawa, Ethiopia.

Received: 30 October 2012 Accepted: 25 November 2013

Published: 1 December 2013

\section{References}

1. Moredun: Controlling Pasteurella Pneumonia in Sheep. 2009. edition of the Sheep farmer www.moredun.org.uk/webfm_send/340.

2. FAO: FAO statistic series. 49th edition. Rome, Italy: FAO; 1995:130.

3. Anon: State of Ethiopians Animal Genetic Resource Country Report. Addis Ababa, Ethiopia: A contribution to the First Report on the State of the World's Animal Genetic Resource. Institute of Biodiversity Conservation (IBC); 2004:74.

4. Anon: Ethiopia livestock sector development projects. Rome, Italy: FAO (Food and Agriculture Organization of the United Nations).Report FAO; 1992. 107/92.

5. Sissay MM: Heminth Parasites of sheep and goals in taster Ethiopia: Epidemlology, and anthelmintic resistance and its management. Uppsala, Sweden: Doctorial thesis, Swedish University of Agricultural seiences; 2007. IJJN 1652-6880, ISBN 978-91-576-7351-0.

6. Herenda D, Chambers P, Ettriqui A, Seneviratna P, Da Silva T: Manual on meat inspection for developing countries. Rome, Italy: FAO; 2000. M-25 ISBN 92-5-103304-103308.

7. Sahni SK: Indian national science academy: Guidelines for care and use of animals in scientific research. New delhi: Bahadur Shah Zafar Marg; 2000. 110 002 and printed at bengal offset works, 335, khajoor road, karol bagh, new delhi 110005.

8. Carter GR: Diagnostic procedures in Veterinary Bacteriology and Mycology. Charles. 4th edition. USA: Thomas; 1984:3-19.

9. Quinn PJ, Carter ME, Markey B, Carter GR: Bacterial pathogens: microscopy, culture and identification. In Clinical Veterinary Microbiology. London, England: Wolfe publishing; 1994:20-60.

10. Sharma SN, Adlakha SC: Text book of veterinary Microbiology. Masjid: Vikas publishing house PVT LTD; 1996.

11. Carter GR: Isolation and identification of bacteria from clinical specimens. In Diagnostic Procedures in Veterinary Bacteriology and Mycology. 4th edition. Edited by Charles C. USA: Thomas; 1984:19-30.

12. Quinn PJ, Carter ME, Markey B, Carter GR: Bacterial Pathogens: Microscopy, Culture, and Identification. In Clinical Veterinary Microbiology. London: Wolfe Publishing; 1994:21-60.

13. MacFadinn JF: Biochemical tests for identification of medical bacteria. 3rd edition. New York: Williams and Wilkins Lippincott. ISBN: 2000:0683,0531805183.

14. NCCLS: National Committee for Clinical Laboratory Standards for antimicrobial disc susceptibility tests. 4th edition. USA: Villonova; 1990.

15. Hendriksen RS: A global Salmonella surveillance and laboratory support project of the World Health Organization. Laboratory Protocols (Isolation of Salmonella) http://www.antimicrobial resistance.dk/data/images/ salmonella2-pdf): 2003:4.

16. Carter JR: Diagnostic procedures in Veterinary Bacteriology and Mycology. 4th edition. Philadelphia: Leafebiger; 1991:367-395.

17. Quinn PJ, Carter ME, Markey B, Carter GR: Clinical Veterinary Microbiology. London: Mosby publish ring; 1999:43-45. 327-394.

18. Clinical and Laboratory Standards Institute (CLSI): Performance Standards for Antimicrobial Susceptibility Testing; Sixteenth Informational Supplement. 940 West Valley Road, Suite 1400, Wayne, Pennsylvania 19087-1898 USA: Clinical and Laboratory Standards Institute; 2006.

19. Eshetu M: Pneumonic Pasteurellosis in sheep slaughtered at Addis Ababa abattoir. Debre-Zeit: DVM thesis, FVM, AAU; 1991.

20. Nurhusein Y: Aerobic bacterial Flora in the respiratory tract of apparently healthy sheep slaughtered in Dessie Abattror, North Eastern Ethiopia. Debre-Zeit: DVM thesis, FVM, AAU; 2005

21. Mohammed R: Bacteriological and histological examination of Pneumonic lungs of small ruminants slaughtered at Hashim Nure export abattoir. Debre-Zeit: DVM thesis, FVM, AAU; 1999:21.

22. Aschalew Z: A study on Pneumonic Pasteurellosis in North Shewa. Debre-Zeit: DVM thesis, AAU, FVM; 1998:59.

23. Tesfaye S: Serological and bacteriological investigation of P.haemolytica serotypes in sheep in the high lands of wollo, North Eastern Ethiopia. Debre-Zeit: DVM thesis, AAU, FVM; 1997:65. 
24. Aiello SE, May A: The Merck Veterinary Mannual. 8th edition. New Jersey. USA: Merck and Co, Inc; 1998:1053-1057.

25. Almeida PFJ, Alves FSJ, Santos LF, Posa JS, De-Almeida PF: Revista de Micro Biological, Volume 17. Brazil; 1986:213-215

26. Gilmour NJ, Gilmour JS: Pasteurellosis of sheep. London: Adlam and Rutter; 1989:223-262

27. Catry B, Decostere A, Schwarz S, Kehrenberg C, de Kruif A, Haesebrouck F: Detection of tetracycline-resistant and susceptible pasteurellaceae in the nasopharynx of loose group housed calves. Vet Res Commun 2006, 30:707-715.

28. Welsh RD, Dye LB, Payton ME, Confer AW: Isolation and Antimicrobial susceptibilities of bacterial pathogens from bovine pneumonia, 1994-2002. J Vet Diagn Invest 2004, 16:426-431.

29. Kaan O, Serpil KK, Tayfun C: Frequency and antibiotic susceptibility of Pasteurella multocida and Mannheimia haemolytica isolates from nasal cavities of cattle. Turk J Vet Anim Sci 2010, 34:91-94.

30. Esra S, Yahya K, Selahattin K: Bacterial examinations in the nasal cavity of apparently healthy and unhealthy Holstein cattle. J Anim \& Vet 2009, 8:2355-2359.

31. Post KW, Cole NA, Raleigh RH: In vitro antimicrobial susceptibility of Pasteurella haemolytica and Pasteurella multocida recovered from cattle with bovine respiratory disease complex. J Vet Diagn Invest 1991, 3:124-126.

32. Quinn PJ, Markey BK, Carter ME, Donnelly WJ, Leonard FC: Veterinary Microbiology and Microbial Diseases. 1st edition. USA: Blackwell Science; 2002:49-52.

doi:10.1186/1746-6148-9-239

Cite this article as: Marru et al:: A study on Ovine pneumonic pasteurellosis: Isolation and Identification of Pasteurellae and their antibiogram susceptibility pattern in Haramaya District, Eastern Hararghe, Ethiopia. BMC Veterinary Research 2013 9:239.

\section{Submit your next manuscript to BioMed Central and take full advantage of:}

- Convenient online submission

- Thorough peer review

- No space constraints or color figure charges

- Immediate publication on acceptance

- Inclusion in PubMed, CAS, Scopus and Google Scholar

- Research which is freely available for redistribution 